\title{
ADMM for Exploiting Structure in MPC Problems
}

\section{Journal Article}

Author(s):

Rey, Felix; Hokayem, Peter; Lygeros, John (D)

Publication date:

2021-05

Permanent link:

https://doi.org/10.3929/ethz-b-000482487

Rights / license:

In Copyright - Non-Commercial Use Permitted

\section{Originally published in:}

IEEE Transactions on Automatic Control 66(5), https://doi.org/10.1109/TAC.2020.3022492 


\title{
ADMM for Exploiting Structure in MPC Problems
}

\author{
Felix Rey, Member, IEEE, Peter Hokayem, Member, IEEE, and John Lygeros, Fellow, IEEE
}

\begin{abstract}
We consider a model predictive control (MPC) setting, where we use the alternating direction method of multipliers (ADMM) to exploit problem structure. We take advantage of interacting components in the controlled system by decomposing its dynamics with virtual subsystems and virtual inputs. We introduce subsystem-individual penalty parameters together with optimal selection techniques. Further, we propose a novel measure of system structure, which we call separation tendency. For a sufficiently structured system, the resulting structure-exploiting method has the following characteristics: $(i)$ its computational complexity scales favorably with the problem size; $(i i)$ it is highly parallelizable; ( $\mathrm{iii}$ ) it is highly adaptable to the problem at hand; and $(i v)$, even for a single-thread implementation, it improves the overall performance. We show a simulation study for cascade systems and compare the new method to conventional ADMM.
\end{abstract}

Index Terms-system structure exploitation, predictive control, alternating direction method of multipliers, distribution

\section{INTRODUCTION}

$\mathbf{I}^{2}$ $\mathrm{N}$ early applications, model predictive control (MPC) [1], [2] was used in large and cost-intensive processes, for example in the chemical industry [3]. In such settings, where the expense of the control hardware in the overall process is small, we can use a generously-sized computation device that is capable of hosting a general-purpose solver. In contrast, the rapid advance of information technology brings MPC-based control techniques to mass production [4], e.g., in automotive industries and consumer electronics. In such large-volume production settings, the pressure on cost-per-unit calls for high efficiency, which we can achieve through tailored hardware and specialized algorithms. We focus on the optimization algorithm, and we use the alternating direction method of multipliers (ADMM) [5], [6], a first-order method for solving convex problems. On the hardware side, we consider embedded platforms, such as field-programmable gate arrays (FPGAs) or application-specific integrated circuits (ASICs). ADMM suits embedded devices as it performs only simple and numerically stable operations [7]. Our goal is to increase the synergy between the algorithm, platform, and problem, by adapting ADMM to fit the controlled system. Hence, instead of pursuing a generalist approach that most off-the-shelve solvers provide, we develop a specialization strategy that improves the performance in a specific MPC setting.

We solve MPC problems that are generally composed of a control objective, system dynamics, and additional state and input constraints. When we conventionally apply ADMM [8],

Manuscript received August xx, 2018; revised xxxx xx, 2019.

This work was supported by ABB Corporate Research under Grant 20171224/01. We thank Michael Cantoni from the University of Melbourne for bringing the well-suited application to cascade systems to our attention.

F. Rey rey* and J. Lygeros lygeros* are with the Automatic Control Laboratory at ETH Zurich, Switzerland, $\star @$ control.ee.ethz.ch.

Peter Hokayem peter.al-hokayem@ch.abb.com is with ABB, Switzerland.

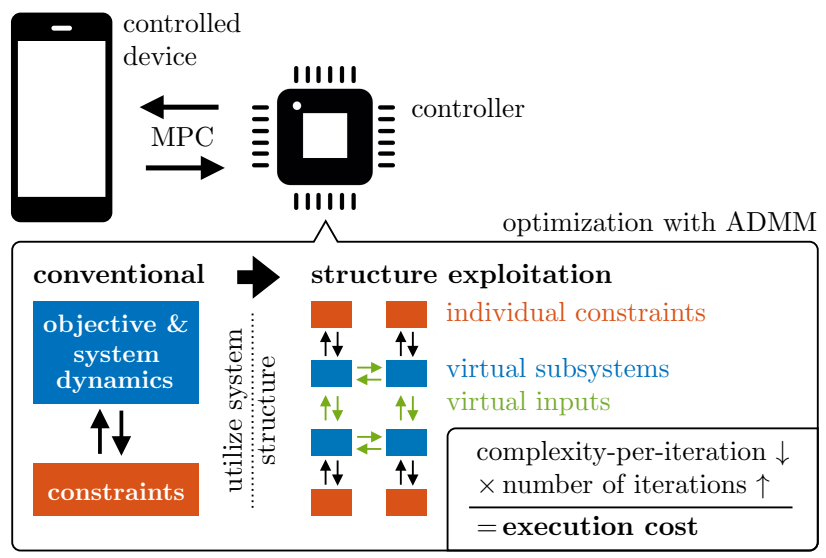

Fig. 1. MPC setup with a controller running structure-exploiting ADMM.

the algorithm mediates between the system dynamics and the additional constraints. We go beyond this formulation by exploiting structure in the controlled system through a decomposition into virtual subsystems, connected through virtual inputs. The tailored algorithm then mediates between all subsystems and reassembles the full system dynamics only in convergence. Fig. 1 illustrates the setup, where we also define the overall cost for executing the algorithm. Our numerical results show that the decomposition typically increases the required number of iterations compared to the conventional application of ADMM. However, the structure-exploiting method reduces the complexity of each iteration and has a large parallelization potential. Overall, if the controlled system is sufficiently structured, structure exploitation reduces the execution cost.

Beside ADMM, Fast Gradient Methods (FGMs) [9] are a common choice for embedded optimization [10]. ADMM often permits more design freedom and can be very efficient for a broad problem range, e.g., for state constraints or convex but not strongly convex objective functions [6]. Examples of problem-specific adaptation through ADMM are [11] for electric vehicle charging; [12] for scenario-based stochastic programs; and [13] for coordinated energy reserve bidding. In these publications, a common theme is the problem separation over an existing coupling constraint. In our approach, we augment the problem with a virtual decomposition before we perform the separation. In [14]-[17], general distributed ADMM formulations are discussed, which do not focus on MPC and do not exploit structure in the problem data. As opposed to potentially non-convergent multi-block extensions [14], [15], our method remains part of the standard ADMM family [6], which guarantees convergence and makes our approach amenable to existing results, e.g., infeasibility detection [18], [19], prescaling [20]-[22], and over-relaxation [6], [23]. Techniques that focus on the MPC setup structure (as opposed to the system 
structure and the ADMM utilization) are also compatible, e.g., move-blocking [24] and warm-starting [22], [24].

Two ADMM formulations [8], [20] are widely used in MPC. For the formulation in [20], the problem needs to be condensed through constraint elimination, which also eliminates structure. With the method in [8], no condensing is required. The formulations also differ in the addressed problem type. In [8], state constraints are possible, and the formulation is particularly suited for easily-projectable constraint sets. We build on this formulation as it preserves the problem structure.

Our method is the result of ongoing research [25], [26]. In [25], we use the basic idea of system structure exploitation for optimization problems that arise from controlling power conversion systems. We generalize the approach to an MPC framework in [26], where we also adopt the name structureexploiting ADMM. In the present work, we advance the overall framework and we present two major novelties: We introduce subsystem-individual penalty parameters with an optimal selection technique, and we propose a novel measure of system structure that we call separation tendency.

Notation: We denote dimensions with matching nonitalic symbols, e.g., $x \in \mathbb{R}^{\mathrm{x}}$. The identity matrix is $I_{n} \in \mathbb{R}^{n \times n}$, and $0_{n \times m}, 1_{n \times m}$ are $n \times m$ matrices with all elements 0 or 1 . We omit the subscripts if the dimension is clear from context. We write $\|x\|_{Q}^{2}$ for $x^{\top} Q x$. We use $\left[A_{i j}\right]$ to concatenate matrices $A_{i j}$ along row $i$ and column $j$, as well as vertical $\left[A_{1 j} ; A_{2 j}\right]$, horizontal $\left[A_{i 1}, A_{i 2}\right]$, and diagonal $\operatorname{diag}\left(A_{11}, A_{22}\right)$ concatenation. We denote a sequence of elements $x_{i}$ with $\left\{x_{i}\right\}_{i=1,2, \ldots}$, where we again may omit the range of $i$.

\section{MPC Formulation AND SySTEM STRUCTURE}

We consider the MPC problem

$$
\begin{array}{rlr}
\min _{\left\{x^{k+1}, u^{k}\right\}} & \sum_{k=1}^{N}\left(\frac{1}{2}\left\|x^{k+1}-r_{x}^{k}\right\|_{Q}^{2}+\frac{1}{2}\left\|u^{k}-r_{u}^{k}\right\|_{R}^{2}\right) \\
\text { s.t. } & x^{k+1}=A x^{k}+B u^{k} & \forall k=1, \ldots, N \\
& \left(x^{k+1}, u^{k}\right) \in \mathcal{X} \times \mathcal{U} & \forall k=1, \ldots, N,
\end{array}
$$

with prediction horizon $N$, state $x^{k} \in R^{\mathrm{x}}$, input $u^{k} \in R^{\mathrm{u}}$, dynamics matrix $A$, input matrix $B$, tracking references $r_{x}^{k}, r_{u}^{k}$, symmetric weights $Q, R$, and constraint sets $\mathcal{X}, \mathcal{U}$. A generalization to time-dependent weights and constraints is straightforward. We use Assumption 1 throughout the paper.

\section{Assumption 1. Problem (1) is convex and feasible.}

Problem (1) is convex if $Q, R$ are positive semidefinite and $\mathcal{X}, \mathcal{U}$ are convex. The problem is feasible if there exist trajectories $\left\{x^{k+1}, u^{k}\right\}_{k=1, \ldots, N}$ that satisfy (1b), (1c). Our approach is particularly suited if a projection onto $\mathcal{X}, \mathcal{U}$ is computationally cheap, as these projections will be used repeatedly.

\section{A. State and Input Partition}

Our method exploits interacting components in the system $(A, B)$. We formalize the presence of such components with the state and input partition $x^{k}=\left[x_{1}^{k} ; \ldots ; x_{M}^{k}\right], u^{k}=$ $\left[u_{1}^{k} ; \ldots ; u_{M}^{k}\right]$. We use $x_{i}^{k} \in \mathbb{R}^{\mathrm{x}_{i}}, u_{i}^{k} \in \mathbb{R}^{\mathrm{u}_{i}}$ with $\mathrm{x}_{i} \in \mathbb{N}_{>0}$, $\mathrm{u}_{i} \in \mathbb{N}_{\geq 0}, \sum_{i} \mathrm{x}_{i}=\mathrm{x}$, and $\sum_{i} \mathrm{u}_{i}=\mathrm{u}$. We assume an state
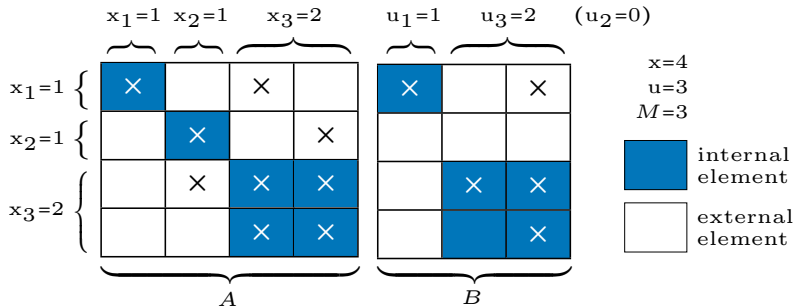

Fig. 2. Illustration of a structured system, where $\times$ is a placeholder for any non-zero element. We choose the partition to best-resemble dense components, indicated by the matrix occupation pattern. A suitable partition leaves few non-zero external elements, while it still decomposes the system.

and input ordering such that only consecutive elements are grouped. Hence, the dimensions $\left\{\mathrm{x}_{i}, \mathrm{u}_{i}\right\}_{i=1, \ldots, M}$ define the partition. In Fig. 2, we show an exemplary component pattern and introduce the notion of internal and external elements. The partition decomposes $A, B$ into submatrices $A_{i j}=\mathbb{R}^{\mathrm{x}_{i} \times \mathrm{x}_{j}}$, $B_{i j}=\mathbb{R}^{\mathrm{x}_{i} \times \mathrm{u}_{j}}$ such that $A=\left[A_{i j}\right], B=\left[B_{i j}\right]$. As $\mathrm{u}_{i} \geq 0$, empty submatrices can appear. Furthermore, we decompose $A, B$ into a sum of internal matrices $\dot{A}, \dot{B}$ and external matrices $\dot{A}, \dot{B}$, where $A=\dot{A}+\dot{A}$ with $\dot{A}=\operatorname{diag}\left(A_{11}, \ldots, A_{M M}\right)$ and $B=\dot{B}+B$ with $\dot{B}=\operatorname{diag}\left(B_{11}, \ldots, B_{M M}\right)$.

Our aim is to partition Problem (1) along the same lines as the system. Towards this, we require the following assumption.

Assumption 2. The partition $\left\{\mathrm{x}_{i}, \mathrm{u}_{i}\right\}$ can be used to decompose (1a) and (1c), i.e., there exist $Q_{i}, R_{i}, \mathcal{X}_{i}, \mathcal{U}_{i}, i=1, \ldots, M$ such that $Q=\operatorname{diag}\left(Q_{1}, \ldots, Q_{M}\right), R=\operatorname{diag}\left(R_{1}, \ldots, R_{M}\right)$, $\mathcal{X}=\left\{x^{k} \mid x_{i}^{k} \in \mathcal{X}_{i} \forall i\right\}$, and $\mathcal{U}=\left\{u^{k} \mid u_{i}^{k} \in \mathcal{U}_{i} \forall i\right\}$.

We say the partition is admissible for (1) if Assumption 2 is satisfied. Any partition is admissible if $Q, R$ are diagonal and $\mathcal{X}, \mathcal{U}$ are separable. Conversely, the trivial partition $M=1$ is admissible for any problem. We can also model constraints that otherwise would prevent admissibility in (1b). If the external part of the partition is sparse, our approach leads to computational benefits. In such a case, we call the respective system structured. We present a better-quantified structure measure in Section IV. A suitable partition can either be found by inspection or with spectral clustering methods [27]-[29], where [29] also provides a suitable state ordering.

\section{B. Virtual Inputs and Subsystems}

By using an admissible partition $\left\{\mathrm{x}_{i}, \mathrm{u}_{i}\right\}$, we rewrite system (1b) as $x^{k+1}=\dot{A} x^{k}+\dot{B} u^{k}+v^{k}$ with the virtual input

$$
v^{k}=\stackrel{A}{A} x^{k}+B u^{k},
$$

which represents the external coupling. As $\dot{A}, \dot{B}$ are blockdiagonal, we can make the decomposition more explicit by rewriting the system as a collection of virtual subsystems

$$
x_{i}^{k+1}=A_{i i} x_{i}^{k}+B_{i i} u_{i}^{k}+v_{i}^{k}, \quad i=1, \ldots, M,
$$

where $v^{k}$ adopts the partition of $x^{k}$. If $A, B$ are sparse, then the virtual input $v_{i}^{k} \in \mathbb{R}^{\mathrm{x}_{i}}$ can take values in a lower dimensional space than suggested by its dimension $\mathrm{x}_{i}$. To make this explicit, we write the components in (2) as

$$
v_{i}^{k}=\sum_{j} A_{i j} x_{j}^{k}+\sum_{j} B_{i j} u_{j}^{k},
$$


where $j \in\{1, \ldots, M\} \backslash\{i\}$. We pick a matrix $W_{i} \in \mathbb{R}^{\mathrm{x}_{i} \times \mathrm{w}_{i}}$ with $\mathrm{w}_{i} \leq \mathrm{x}_{i}$ such that its columns form a range space basis for the concatenated matrix

$$
\left[\left\{A_{i j}, B_{i j}\right\}_{j \in\{1, \ldots, M\} \backslash\{i\}}\right] \in \mathbb{R}^{\mathrm{x}_{i} \times\left(\mathrm{x}+\mathrm{u}-\mathrm{x}_{i}-\mathrm{u}_{i}\right)},
$$

which we obtain by writing (4) as a single matrix-vector operation. Hence, $\mathrm{w}_{i}$ is the row rank of (5), and the range space of $W_{i}$ contains all values that $v_{i}^{k}$ can attain. We then introduce the dimension-reduced virtual input $w_{i}^{k} \in \mathbb{R}^{\mathrm{w}_{i}}$ by replacing $v_{i}^{k}$ with $W_{i} w_{i}^{k}$.

\section{Partitioned Problem}

By using an admissible partition $\left\{\mathrm{x}_{i}, \mathrm{u}_{i}\right\}$, we write (1) as

$$
\begin{array}{rlr}
\min _{\left\{x^{k+1}, u^{k}, w^{k}\right\}} & \sum_{i, k=1}^{M, N}\left(\frac{1}{2}\left\|x_{i}^{k+1}-r_{x_{i}}^{k}\right\|_{Q_{i}}^{2}+\frac{1}{2}\left\|u_{i}^{k}-r_{u_{i}}^{k}\right\|_{R_{i}}^{2}\right) \\
\text { s.t. } & x_{i}^{k+1}=A_{i i} x_{i}^{k}+B_{i i} u_{i}^{k}+W_{i} w_{i}^{k} & \forall(i, k) \\
& \left(x_{i}^{k+1}, u_{i}^{k}\right) \in \mathcal{X}_{i} \times \mathcal{U}_{i} & \forall(i, k) \\
& W w^{k}=A x^{k}+B u^{k} & \forall k,
\end{array}
$$

where $w^{k}=\left[w_{1}^{k} ; \ldots ; w_{M}^{k}\right] \in \mathbb{R}^{\mathrm{w}}$ and $W=\operatorname{diag}\left(W_{1}, \ldots, W_{M}\right)$. Furthermore, with the stacked variables $y_{i}^{k}=\left[u_{i}^{k} ; w_{i}^{k} ; x_{i}^{k+1}\right]$, $y_{i}=\left[y_{i}^{1} ; \ldots ; y_{i}^{N}\right] \in \mathbb{R}^{y_{i}}$, and $y=\left[y_{1} ; \ldots ; y_{M}\right] \in \mathbb{R}^{\mathrm{y}}$, we obtain

$$
\begin{aligned}
& \min _{y} \sum_{i=1}^{M}\left(\frac{1}{2} y_{i}^{\top} \mathcal{Q}_{i} y_{i}+q_{i}^{\top} y_{i}+K_{i}\right) \\
& \text { s.t. } \quad C_{i} y_{i}=c_{i} \quad \forall i \\
& y_{i} \in \mathcal{Y}_{i} \quad \forall i \\
& D y=d \text {. }
\end{aligned}
$$

We list the definitions of $\mathcal{Q}_{i}, q_{i}, K_{i}, C_{i}, c_{i}, \mathcal{Y}_{i}, D$, and $d$ in Appendix A-A. Problem (7) is equivalent to Problem (1).

\section{Conventional ADMM}

Our approach extends the conventional ADMM formulation in [8], which considers the unpartitioned problem

$$
\begin{array}{cl}
\min _{y} & \frac{1}{2} y^{\top} \mathcal{Q} y+q^{\top} y+K \\
\text { s.t. } & C y=c \\
& y \in \mathcal{Y} .
\end{array}
$$

For $M=1$, Problem (8) is equivalent to Problem (7). As in [8], we rewrite (8c) with $y=\zeta, \zeta \in \mathcal{Y}$, where $\zeta$ is a duplicate of $y$. As shown in Algorithm 1, ADMM then addresses the parts depending on $y$ and $\zeta$ alternatingly. We use an orthogonal projection $\Pi$ as in [8], [30], a scaled Lagrange multiplier $\lambda$ which is associated to the constraint $y=\zeta$, and a user-defined penalty parameter $\rho$ that influences the convergence speed [6]. It is shown in [8] that Algorithm 1 with $\rho>0$ converges to a fixed point that is optimal for (8). Suitable initialization and termination techniques are discussed in [6], and the behavior for infeasible problems is discussed in [19], [31].

\section{StRucture-EXPLOITING ADMM}

The structure-exploiting algorithm utilizes the problem partition and therefore takes advantage of the system structure.

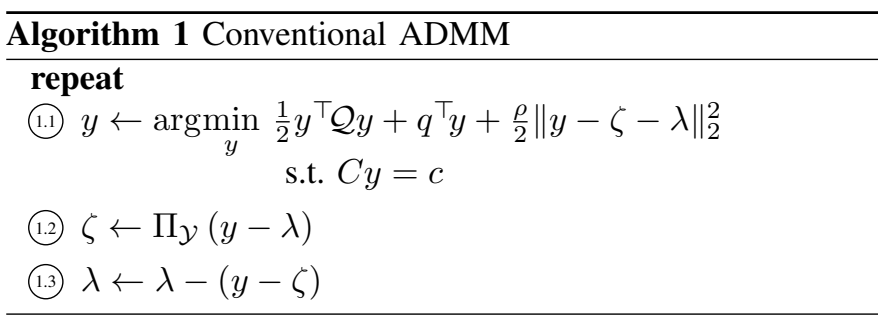

\section{A. Main Algorithm}

To solve Problem (7) with $M>1$, we add (additional to $\zeta$ ) the second duplicate $\epsilon$. Equivalent to (7), we obtain

$$
\begin{aligned}
& \min _{y, \zeta, \epsilon} \sum_{i=1}^{M}\left(\frac{1}{2} y_{i}^{\top} \mathcal{Q}_{i} y_{i}+q_{i}^{\top} y_{i}+K_{i}\right) \text { (individual objec- } \\
& \text { s.t. } C_{i} y_{i}=c_{i} \quad \forall i \\
& \zeta_{i} \in \mathcal{Y}_{i} \quad \forall i \quad \text { (individual constraints) } \\
& D \epsilon=d \\
& y=\zeta=\epsilon \text {. }
\end{aligned}
$$

We show the resulting structure-exploiting ADMM formulation in Algorithm 2, and we show a detailed derivation in Appendix A-B. We use the Lagrange multipliers $\lambda_{\zeta}, \lambda_{\epsilon}$, which have the same size and partition as $y$. Further, we introduce subsystem-individual penalty parameters $\rho_{i}>0$, a balancing parameter $\beta \in(0,1]$, and the modified projection operation

$$
\bar{\Pi}_{D \epsilon=d}(\cdot)=E_{\epsilon}^{-1 / 2} \Pi_{D E_{\epsilon}^{-1 / 2} \epsilon=d}\left(E_{\epsilon}^{1 / 2} \cdot\right),
$$

where $E_{\epsilon}=(1-\beta) \operatorname{diag}\left(\rho_{1} I_{\mathrm{y}_{1}}, \ldots, \rho_{M} I_{\mathrm{y}_{M}}\right)$. In (2.1), $\beta$ adjusts the balance between the regularization terms and therefore can affect the convergence speed. We discuss its choice in Section III-C. Proposition 1, proven in Appendix A-B, describes how we use Algorithm 2 to solve the original problem (1).

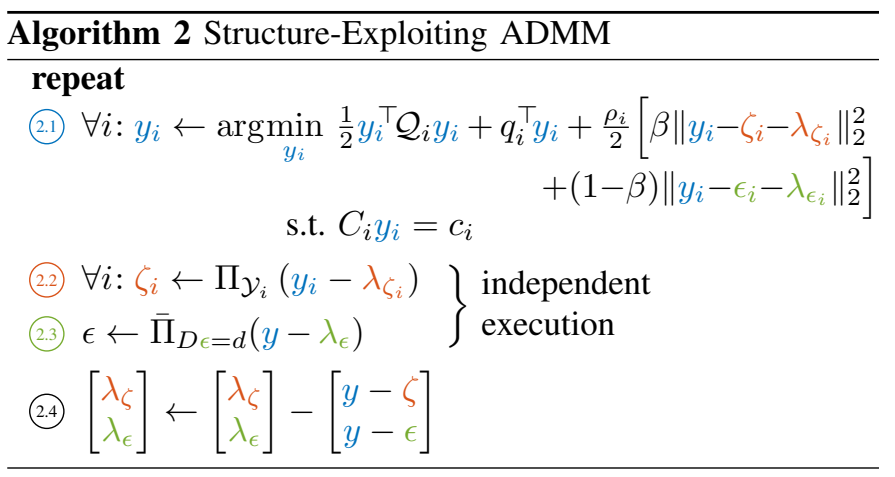

\section{Proposition 1.}

(i) If $\beta \in(0,1)$ and $\rho_{i}>0$ for all $i$, Algorithm 2 converges to a fixed point $y^{\star}=\zeta^{\star}=\epsilon^{\star}$ that is optimal for (9) and (1).

(ii) If $M=\beta=1$ and $\rho_{1}=\rho$, Algorithm 2 reduces to Algorithm 1.

In Appendix A-B, we introduce $\beta$ and $\rho_{i}$ through a metric selection technique [18], [23], which makes Algorithm 2 part of the standard ADMM family. The convergence statement in Proposition 1 leaves out the case of $\beta=1, M>1$, for which the algorithm is unsuited as it solves (7) without (7d). 
TABLE I

COMPLEXITIES FOR EACH STEP IN ALGORITHM 2



Fig. 3. Illustration of the composition of Algorithm 2. Each step exploits different types of structure, which improves the computational efficiency.

TABLE II

COMPLEXITY-PER-ITERATION FOR ALGORITHM 2

\begin{tabular}{llll}
\hline & use case & threads & complexity of the longest thread $\mathcal{O}(\cdot)$ \\
\hline$\# 1$ & $M=1$, box & 1 & $N \mathrm{x}^{2}+N \mathrm{x}$ \\
$\# 2$ & box & 1 & $N\left(M \max _{i} \mathrm{x}_{i}^{2}+M \max _{i} \mathrm{x}_{i}+\mathrm{w}^{2}\right)$ \\
$\# 3$ & box & $2 M N$ & $N \max _{i} \mathrm{x}_{i}^{2}+\max \left\{\max _{i} \mathrm{x}_{i}, \mathrm{w}^{2}\right\}$ \\
$\# 4$ & box, out-1 & 1 & $M N\left(\max _{i} \mathrm{x}_{i}^{2}+\max _{i} \mathrm{x}_{i}+\max _{i} \mathrm{w}_{i}^{2}\right)$ \\
$\# 5$ & box, out-1 & $2 M N$ & $N \max _{i} \mathrm{x}_{i}^{2}+\max _{i}\left\{\mathrm{x}_{i}, \mathrm{w}_{i}^{2}\right\}$
\end{tabular}

\section{B. Efficient Implementation and Computational Complexity}

In this section, show the large parallelization potential of our method. Step (2.1) is an equality-constrained quadratic program. With the Schur complement method in [32, Sec. 16.2], we obtain the closed-form solution

$y_{i} \leftarrow \mathcal{M}_{i}\left(-\frac{1}{\rho_{i}} q_{i}+\beta\left(\lambda_{\zeta_{i}}+\zeta_{i}\right)+(1-\beta)\left(\lambda_{\epsilon_{i}}+\epsilon_{i}\right)\right)+\mathcal{N}_{i} c_{i}$,

where $\mathcal{N}_{i}=\mathcal{P}_{i} C_{i}^{\top}\left(C_{i} \mathcal{P}_{i} C_{i}^{\top}\right)^{-1}, \mathcal{M}_{i}=\left(I-\mathcal{N}_{i} C_{i}\right) \mathcal{P}_{i}$, and $\mathcal{P}_{i}=\rho_{i}\left(\mathcal{Q}_{i}+\rho_{i} I\right)^{-1}$. An efficient implementation of (11) exploits the MPC multistage structure [33], i.e., it exploits the fact that $C_{i}$ is banded (see Appendix A-C for details).

Similarly, the modified affine projection in (2.3) can be written as an equality-constrained quadratic program and solved by $\epsilon \leftarrow \mathcal{D}\left(y-\lambda_{\epsilon}\right)+\mathcal{E} d$, where $\mathcal{E}=E_{\epsilon}^{-1} D^{\top}\left(D E_{\epsilon}^{-1} D^{\top}\right)^{-1}$ and $\mathcal{D}=I-\mathcal{E} D$. Further, we consider a permutation matrix $P$ that sorts $y$ for time, i.e., $P y=\bar{y}=\left[\bar{y}^{1} ; \ldots ; \bar{y}^{N+1}\right]$ with $\bar{y}^{1}=\left[u^{1} ; w^{1}\right], \bar{y}^{k=2, \ldots, N}=\left[x^{k} ; u^{k} ; w^{k}\right]$, and $\bar{y}^{N+1}=x^{N+1}$. In the permuted coordinates, we use $\overline{\mathcal{D}}=P \mathcal{D} P^{\top}$ and $\overline{\mathcal{E}}=P \mathcal{E}$, which are then block-diagonal. Hence, we can solve (2.3) with

$$
\forall k=1, \ldots, N+1: \quad \bar{\epsilon}^{k} \leftarrow \overline{\mathcal{D}}^{k}\left(\bar{y}^{k}-\bar{\lambda}_{\epsilon}^{k}\right)+\overline{\mathcal{E}}^{k} d^{k},
$$

where $\bar{\lambda}_{\epsilon}=P \lambda_{\epsilon}, \bar{\epsilon}=P \epsilon$, and the partition in $k$ matches $\bar{y}^{1}, \ldots, \bar{y}^{N+1}$. A special case is when each subsystem influences
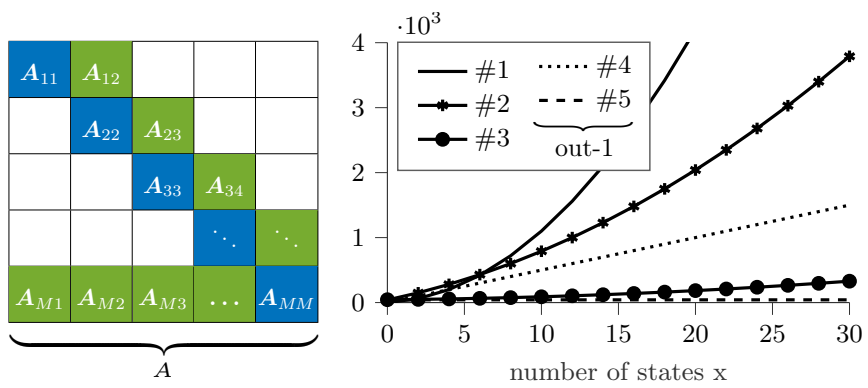

Fig. 4. Left: Occupation pattern of an example dynamics matrix. Right: Complexities-per-iteration in Table II for a growing system size.

at most one other subsystem through a virtual input. In this case, $\overline{\mathcal{D}}^{k}$ and $\overline{\mathcal{E}}^{k}$ decompose further along $\mathrm{w}_{1}, \ldots, \mathrm{w}_{M}$, as the virtual inputs are independent of each other. Consequently, (12) can be executed with $M N$ parallel threads.

Table I shows the computational complexities of each algorithm step, justifications are shown in Appendix A-C. The Landau symbol $\mathcal{O}$ describes the order of required scalar multiplications and additions. We denote the computational costs for projecting onto $\mathcal{X}_{i}, \mathcal{U}_{i}$ with $\mathrm{c}\left(\Pi_{\mathcal{X}_{i}}\right), \mathrm{c}\left(\Pi_{\mathcal{U}_{i}}\right)$. We consider two special cases: 'box' denotes the case where $\mathcal{X}_{i}, \mathcal{U}_{i}$ are easily-projectable box constraints; and 'out-1' denotes the case where each subsystem affects at most one virtual input. Aside from the structure in the algorithm steps, the composition of Algorithm 2 also offers a large potential for parallelization:

- 2.1. and (2.2) are separate for each subsystem

- 2.2. consists of $M N$ separate projections onto $\mathcal{X}_{i}, \mathcal{U}_{i}$

- 23) separates for each step along the prediction interval

- 2.2. and (2.3) are independent from each other.

We summarize the composition in Fig. 3. Table II shows the complexity-per-iteration of Algorithm 2. The case $M=1$, independent of $\beta$, has the same complexity as conventional ADMM. We consider an example system to illustrate the complexities in Fig. 4. In Table II, we see that the obtained values follow from the problem parameters $M, N,\left\{\mathrm{x}_{i}, \mathrm{w}_{i}\right\}$, the number of threads, and the use case. We use $A_{i j} \in \mathbb{R}^{2 \times 2}$ for all $i, j$. We assume that each $A_{i, i+1}$ has rank 1, i.e., $w_{i}=1$ for $i \neq M$. For the last component, we assume $\mathrm{w}_{M}=2$. We use $N=10$ and we consider systems with 1-15 diagonal components, i.e., $\mathrm{x} \in\{2,4,6, \ldots, 30\}$. In Fig. 4 on the right, we show the corresponding complexities. The cases \#4,5 only apply if $A_{i, i+1}=0$ or $A_{M, i}=0$, i.e., the case 'out-1' applies. We see that structure exploitation (\#2-5) compares favorably to conventional ADMM (\#1), even for singlethread implementations $(\# 2,4)$. We also see that the benefit from structure exploitation grows with the system size. As presented in Fig. 1, the complexity-per-iteration only defines a part of the overall execution cost. We analyze the remaining part, namely the required number of iterations, in Section IV.

\section{Parameter Choice}

We denote the smallest and largest eigenvalues of a matrix with eig $_{\min }, \mathrm{eig}_{\max }$. We consider an orthonormal null space basis for $C_{i} \in \mathbb{R}^{N \mathrm{x}_{i} \times \mathrm{y}_{i}}$, and use the basis vectors as columns in $Z_{i} \in \mathbb{R}^{\mathrm{y}_{i} \times N \mathrm{x}_{i}}$, which leads to $C_{i} Z_{i}=0$ and $Z_{i}^{\top} Z_{i}=I_{N \mathrm{x}_{i}}$. 
Proposition 2. We assume that $Z_{i}^{\top} \mathcal{Q}_{i} Z_{i}$ is positive definite and $\beta \in(0,1)$. The optimal penalty parameters for improving the worst-case convergence rate of Algorithm 2 are

$$
\rho_{i}^{\star}=\sqrt{\operatorname{eig}_{\min }\left(Z_{i}^{\top} \mathcal{Q}_{i} Z_{i}\right) \operatorname{eig}_{\max }\left(Z_{i}^{\top} \mathcal{Q}_{i} Z_{i}\right)},
$$

where $i=1, \ldots, M$.

We show a proof of Proposition 2 in Appendix A-D, and we provide additional details and technical considerations in [34]. The proof also shows that $\beta$ is canceled out when we derive $\rho_{i}^{\star}$, i.e., the parameters can be chosen independently. Proposition 2 suggests that individual penalty parameters are indeed useful, as their optimal choice is different from making them all the same. The optimal parameters (13) are valid for any quadratic program of type (7). For MPC problems in particular, and similar as for comparable results [8], [19], [23], we observe that we often can improve the practical performance further by increasing the penalties above $\rho_{i}^{\star}$, which places an additional weight on the regularization terms in (2.1.)

As noted before, $\beta \in(0,1]$ adjusts the regularization balance in (2.1.). The two regularization terms are equally weighted for $\beta=\frac{1}{2}$. By increasing $\beta$ we emphasize the influence of $\left(\zeta_{i}, \lambda_{\zeta_{i}}\right)$ over $\left(\epsilon_{i}, \lambda_{\epsilon_{i}}\right)$, and vice versa. We can also adapt $\beta$ during the algorithm iteration, similar to $\rho$ in [6, Sec. 3.4.1]. However, our numerical results suggest that the effect of $\beta$ is small for the cases considered below. For this reason, we restrict our attention to the nominal values $\beta \in\left\{\frac{1}{2}, 1\right\}$ in the sequel. For $\beta=\frac{1}{2}$ and $M>1$, we speak of structure-exploiting ADMM. With $\beta=M=1$, we refer to conventional ADMM.

\section{Separation Tendency}

We derive a quantitative measure of system structure, called the separation tendency. We use the separation tendency as a heuristic indicator for the required number of algorithm iterations relative to conventional ADMM. In combination with the complexity results in Table II, this makes it possible to anticipate the execution cost of structure exploiting ADMM.

\section{A. System Flow and Link Usage}

Our goal is to quantify the interaction between system components. We first consider the unpartitioned $\Delta$-system

$$
\Delta x^{k+1}=A \Delta x^{k}+B \Delta u^{k},
$$

where $\Delta x^{k}=x^{k}-x^{k-1}, \Delta u^{k}=u^{k}-u^{k-1}, k \geq 0$, and we use the convention $\left(x^{-1}, u^{-1}\right)=(0,0)$. The $\Delta$-system describes the changes in the original system (1b).

Definition 1. The system flow $\Phi^{k}=\left[\Phi_{A}^{k}, \Phi_{B}^{k}\right] \in \mathbb{R}^{\mathrm{x} \times(\mathrm{x}+\mathrm{u})}$ is composed of the state-to-state and input-to-state flow

$$
\begin{aligned}
& \Phi_{A}^{k}=A \operatorname{diag}\left(\Delta x^{k}\right) \in \mathbb{R}^{\mathrm{x} \times x} \\
& \Phi_{B}^{k}=B \operatorname{diag}\left(\Delta u^{k}\right) \in \mathbb{R}^{\mathrm{x} \times u} .
\end{aligned}
$$

We use the operation $A \operatorname{diag}(\Delta x) \in \mathbb{R}^{\mathrm{x} \times \mathrm{x}}$ to analyze system-internal effects. With $A \operatorname{diag}(\Delta x) 1_{\mathrm{x} \times 1}=A \Delta x$, it becomes clear that we can understand $A \operatorname{diag}(\Delta x)$ as an intermediate step that leads to the matrix-vector product $A \Delta x$. By following this relation, we obtain

$$
x^{k+1}=x^{k}+\Phi^{k} 1_{(\mathrm{x}+\mathrm{u}) \times 1},
$$

which clarifies that the system flow $\Phi^{k}$ describes the state transition. A central characteristic of $\Phi^{k}$ is that it details the transition contribution for each of the $\mathrm{x}^{2}$ state-to-state links and $\mathrm{x} \cdot \mathrm{u}$ input-to-state links.

The link usage, which we introduce next, measures the flow that moves through each system link in response to a unit input impulse $\delta^{k}$ with $\delta^{k=0}=1$ and $\delta^{k \neq 0}=0$.

Definition 2. The link usage $\Gamma=\left[\Gamma_{A}, \Gamma_{B}\right] \in \mathbb{R}^{\mathrm{x} \times(\mathrm{x}+\mathrm{u})}$ is assembled element-wise with

$$
\Gamma_{i j}=\left(\sum_{k=0}^{\infty}\left|\Phi_{i j}^{k}\right|^{2}\right)^{1 / 2} \in \mathbb{R},
$$

where $\Phi^{k}=\left[\Phi_{i j}^{k}\right], \Gamma=\left[\Gamma_{i j}\right]$, and the sequence $\left\{\Phi^{k}\right\}$ results from $u^{k}=\delta^{k}$ and $x^{0}=0$.

The link usage $\Gamma$ analyzes the system flow over time by using an element-wise $\mathcal{L}_{2}$ norm [35, Chapter 2]. If $\Gamma_{i j}$ is large, then the respective system link is used intensively.

\section{B. Separation Tendency}

The separation tendency compares the link usage for internal and external elements, which provides a relative measure for the concentration of flow inside and outside of virtual subsystems. In contrast to the previous concepts, the separation tendency depends on the system and its partition.

Definition 3. The separation tendency $s \in \mathbb{R}$ is defined by

$$
\begin{aligned}
s_{i} & =\frac{\frac{1}{\# \text { int }_{i}} \sum_{j} \dot{\Gamma}_{i j}}{\frac{1}{\# \text { int }_{i}} \sum_{j} \dot{\Gamma}_{i j}+\frac{1}{\# \operatorname{ext}_{i}} \sum_{j} \Gamma_{i j}^{\mathrm{a}}} \\
s & =\frac{1}{\mathrm{x}} \sum_{i=1}^{\mathrm{x}} s_{i},
\end{aligned}
$$

where $\Gamma=\dot{\Gamma}+\dot{\Gamma}=\left[\dot{\Gamma}_{A}, \dot{\Gamma}_{B}\right]+\left[\vec{\Gamma}_{A}, \dot{\Gamma}_{B}\right]$ is an internal-external decomposition, and \# $\mathrm{int}_{i}$, \# $\mathrm{ext}_{i}$ are the numbers of internal and external elements in the $i$-th row of $\Gamma$.

If the separation tendency $s$ is large, then internal links predominantly influence the states, which signals a clear subsystem separation. If the separation tendency is small, then the system states are dominated by external flow, which signals that the chosen partition is unsuited.

\section{Proposition 3.}

(i) $s$ exists if the system $(A, B)$ is controllable, and $A$ is semi-convergent, i.e., $\lim _{k \rightarrow \infty} A^{k}$ exists.

(ii) If $s$ exists, then $0 \leq s \leq 1$.

(iii) $s$ is invariant to diagonal state and input transformations.

We show a proof of Proposition 3 in Appendix A-E. We use semi-convergence, which is a weaker condition than asymptotic stability, but stronger than marginal stability. The existence of the separation tendency $s$ implies that the link usage $\Gamma$ is finite and does not contain zero rows, which prevents that the denominator in (18a) becomes zero. Property (iii) makes clear that $s$ is unaffected by state and input transformations $[x ; u]=T[\bar{x} ; \bar{u}]$, where $T$ is an invertible and diagonal matrix. This property is important as it makes the separation tendency invariant to simple state and input scaling. 


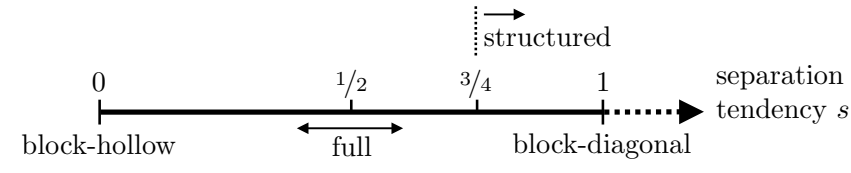

Fig. 5. Empirical characterization of the separation tendency $s \in[0,1]$.

It is easy to show that $s=0$ for block-hollow systems and $s=1$ for block-diagonal systems. Full systems are placed in-between with $s \approx 0.5$. Fig. 5 illustrates the range of $s$. We call a system structured if it can be partitioned with a large separation tendency. An empirically reasonable threshold between structured and unstructured systems is $s=\frac{3}{4}$.

Example 1. We consider the full system

$$
x^{k+1}=\underbrace{\left[\begin{array}{ll}
1 / 2 & 1 / 2 \\
1 / 2 & 1 / 2
\end{array}\right]}_{A} x^{k}+\underbrace{\left[\begin{array}{l}
1 \\
1
\end{array}\right]}_{B} u^{k}
$$

and a partition with $\left\{\mathrm{x}_{i}\right\}=\{1,1\}$ and $\left\{\mathrm{u}_{i}\right\}=\{1,0\}$. We observe that the system matrix is semi-convergent (it even is idempotent, i.e., $(A)^{k}=A$ ). The system is not controllable, therefore $s$ is not guaranteed to exist a-priori. For an input impulse $\delta^{k}$, the system flow and the link usage become

$$
\begin{aligned}
\Phi^{k} & =\delta^{k} \frac{1}{2}\left[\begin{array}{lll}
0 & 0 & 1 \\
0 & 0 & 1
\end{array}\right]+\delta^{k-1} \frac{1}{2}\left[\begin{array}{ccc}
1 & 1 & -1 \\
1 & 1 & -1
\end{array}\right] \\
\Gamma & =\left[\begin{array}{lll}
1 / 2 & 1 / 2 & \sqrt{2} \\
1 / 2 & 1 / 2 & \sqrt{2}
\end{array}\right],
\end{aligned}
$$

where we shade the link usage according to the partition. As all elements in $\Gamma$ are non-zero and finite, the separation tendency exists. We obtain $s=\frac{1}{2}$, which suggests that the considered partitioned system is not suited for structure exploitation.

\section{Algorithm Performance Indication}

We show that $s$ is an indicator for the performance of system structure exploitation. More specifically, we collect empirical evidence that $s$ relates to the growth in required algorithm iterations when we switch from conventional to structureexploiting ADMM. We consider systems in six categories: full, sparse, lower-triangular, banded, lower-banded, and startopology; as described in [34]. Additionally, we consider the dimensions $\mathrm{x} \in\{5,10,20,40\}$, which result in 24 combinations. For each combination, we generate twenty pairs of system matrices, which leads to a test set of 480 systems. For each system, we then generate twenty problems of type (1). For simplicity, and as $s$ only depends on the partitioned system, we set $\mathcal{X}=\mathbb{R}^{\mathrm{x}} \mathcal{U}=\mathbb{R}^{\mathrm{u}}$. We solve the final 9600 problems with conventional and structure-exploiting ADMM. For the structure-exploiting case, we choose a partition that fits to the problem type and dimension as described in [34]. For the penalty parameters, we set $\rho=\rho_{i}=1$. We measure the number of iterations that are necessary to converge within a certain accuracy of a precomputed solution. We then compute the iteration increase factor when we switch between algorithms, and we average this factor over each system's twenty initial conditions. Fig. 6 illustrates the result. The key observation

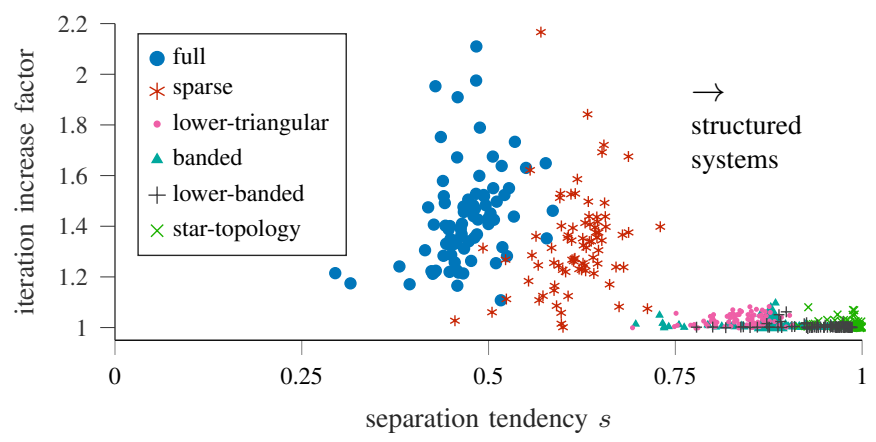

Fig. 6. Iteration increase over the separation tendency. Each of the 480 dots represents an example system. For each system, we generate twenty problem instances, run both algorithms, and depict the average increase.

is that a large separation tendency indicates a low iteration increase. Combined with the complexity results in Table II, this allows us to assess the algorithm performance as defined in Fig. 1, particularly without having to implement and benchmark the algorithm first.

\section{Related Concepts and System Properties}

Several quantities that are used in the literature are related to $\Phi, \Gamma$, and $s$. Spectral clustering methods [27]-[29] interpret the system as a weighted graph, where the elements of $[A, B]$ determine the edge weights. These methods can be used to determine a system partition by cutting possibly low-weighted edges. In contrast to the static weights $[A, B]$, we use impulseresponse-based dynamical links for $\Gamma$. We can assess the value of this dynamical concept by redrawing Fig. 6 while we use $[A, B]$ instead of $\Gamma$ to obtain $s$. We then observe that this static version of $s$ is significantly less indicative for the algorithm iteration growth. Furthermore, clustering methods are sensitive to diagonal state and input transformations.

The similarity of $A, B$ to block-diagonal matrices can be an intuitive structure measure as it directly relates to the sparsity of $A, B$. In [36], a range of diagonality measures is discussed; generalizations to block-diagonality are straightforward. Same as before, these measures ignore dynamic interaction, are less indicative for the iteration growth, and are sensitive to diagonal state and input transformations.

The computation of $\Gamma$ is similar to the computation of the $\mathcal{H}_{2}$ system norm, where the $\mathcal{L}_{2}$ norm is applied to the system's impulse response [37, Eq. (2.37)], [38, Eq. (2.167)]. Two main differences separate the concepts. First, $\Gamma$ is based on the $\Delta$-system, which makes it finite for a wider range of cases. Second, $\Gamma$ is matrix-valued, which underlines the focus on the system-internal state-to-state and input-to-state links. In contrast, the $\mathcal{H}_{2}$ norm is scalar-valued, even if we use the full state vector as system output [37, Eq. (2.37)].

Another related concept is the balanced realization [39, Sec. 4.2], which is a state space system representation with identical and diagonal controllability and observability Gramians. The diagonal elements then quantify the influence of each state on the input-output behavior. The concept resembles $s$ in its quantitative description of system-internal relations. However, $s$ is based on $\Phi$ with $\mathrm{x}^{2}+$ xu elements, while the 
TABLE III

COST-PER-ITERATION OF ALGORITHM 2 FOR THE CASCADE SYSTEM (20)

\begin{tabular}{|c|c|c|c|c|c|}
\hline & ADMM-type & $M$ & threads & cost & $\operatorname{cost} /(i)$ \\
\hline & & 1 & 1 & 277676 & $100 \%$ \\
\hline (ii) & structu & $S$ & 1 & 50220 & $18.09 \%$ \\
\hline (iii) & structure-exploiting & $S$ & $2 M N$ & 3125 & $1.13 \%$ \\
\hline
\end{tabular}

Gramians only have x diagonal elements. Hence, $\Phi$ analyzes the system with a higher resolution. Aside from that, another distinction is that $\Phi$ (and therefore $s$ ) focuses on the state transition, while the Gramians relate to the input-output behavior.

\section{Simulation Study}

We apply structure-exploiting ADMM to a cascade system, where our method leads to a substantial benefit. We also show a negative example of an unstructured system. More positive examples can be found in [25], [26].

\section{A. Cascade System}

A cascade system [40] is characterized by a lower blockbanded dynamics matrix and a block-diagonal input matrix

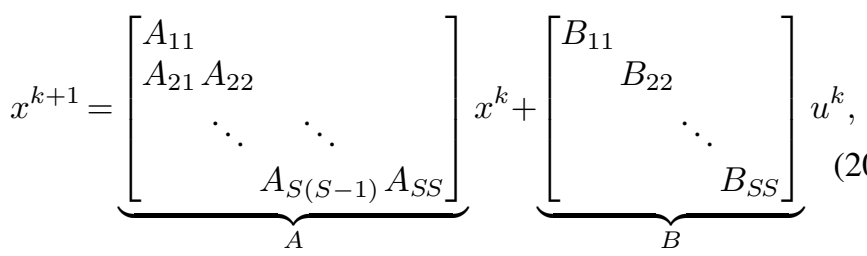

where we use the stages $i=1, \ldots, S$, each with a dynamics matrix $A_{i i}$, input matrix $B_{i i}$, and coupling matrix $A_{i(i-1)}$. Cascade systems are used for irrigation and drainage networks [41], [42], hydro-power systems [43], and vehicle platoon control [44]. In [40], an interior point method is developed, where the iteration complexity scales linearly in $S$ and cubically in $N$, albeit also typically requiring less iterations [25]. For structure-exploiting ADMM, we associate each stage to a virtual subsystem, i.e., $M=S$. Due to the simple subsystem topology, the complexity results \#4,5 in Table II apply. Hence, for a single-thread implementation, the iteration complexity scales linearly in $S$ and $N$. Further, when we use parallel computation, the complexity becomes constant in $S$.

We consider $S=20$ stages, each with $\mathrm{x}_{i}=6$ states and $\mathrm{u}_{i}=1$ input, resulting in a cascade system with $\mathrm{x}=120$ states and $\mathrm{u}=20$ inputs. The system matrices are randomly generated as in [34]. The stage coupling $A_{i(i-1)}$ has a rank equal to 1 . We use (1) with $N=5$ and box constraints. Table III shows estimates for the cost-per-iteration of Algorithm 2 in different situations. In contrast to the analytical bounds in Table II, we obtain the computational costs by counting the scalar additions and multiplications in an actual implementation. This counting strategy is more precise than complexity bounds and takes the remaining matrix sparsity into account. As opposed to timing measurements, it is also less hardware-dependent. For the parallel implementation (iii), we count the operations in the longest thread. We do not account for memory access or data exchange operations,

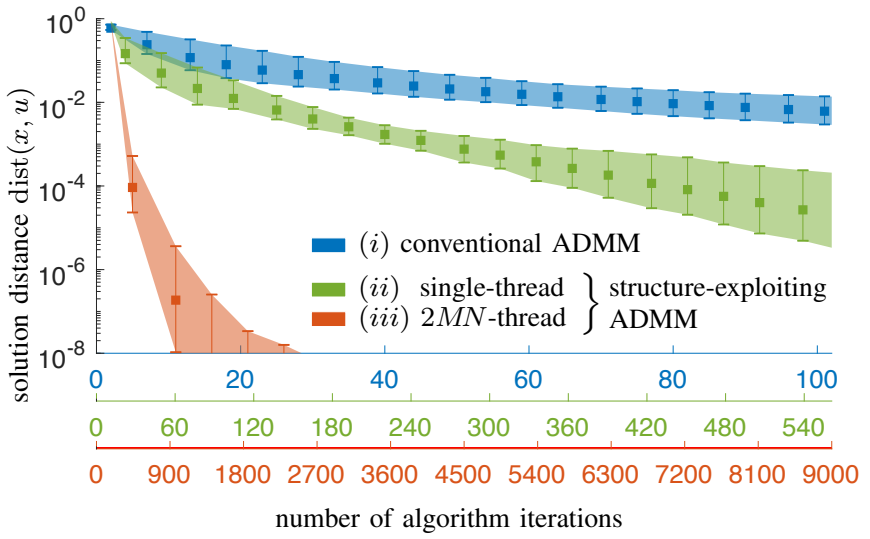

Fig. 7. Convergence of Algorithm 2 for the cascade system (20). The markers indicate the geometric mean over 200 control scenarios; the illustrated range includes $80 \%$ of all scenarios, excluding the best and worst $10 \%$.

which is justified for many FPGA-type implementations where such operations can be hard-coded. We see that structure exploitation significantly reduces the computational cost.

By using the methods in Section IV, we obtain $s=0.975$ for the cascade system. We assess the required number of algorithm iterations by applying $(i)$ - (iii) in a range of control situations. More precisely, we generate 200 feasible instances of (1) as described in [34]. We compute $\rho_{i}^{\star}$ for each subsystem, and we increase the penalty parameters with a factor of 90 for improving the MPC performance as discussed in Section III-C. We then analyze the convergence to a precomputed nonzero solution $\left(x^{\star}, u^{\star}\right)$ with

$$
\operatorname{dist}(x, u)=\left\|[x ; u]-\left[x^{\star} ; u^{\star}\right]\right\|_{2}^{2} /\left\|\left[x^{\star} ; u^{\star}\right]\right\|_{2}^{2},
$$

where $(x, u)$ is the current estimate, extracted after each iteration. We rely on free licenses for Yalmip [45] and Gurobi [46]. In Fig. 7, we show the growing solution accuracy with the number of performed iterations as a statistic over the 200 problem instances. We compare the overall performance of $(i)$ - (iii) by using three different horizontal axes, scaled with the computational costs in Table III. For a given point along these axes, we see how many iterations each method can perform while they use the same number of sequential scalar operations. We observe that for the cascade system, the exploitation of system structure results in faster convergence, even for a single-thread implementation. When we use the full parallelization potential, high performance is possible. By sequentially combining previously parallel threads, it is also possible to obtain implementations that perform between $(i i)$ and (iii) with less than $2 M N$ threads.

\section{B. Unstructured System}

We consider the system from Example 1 with $\left\{\mathrm{x}_{i}\right\}=\{1,1\}$ and $\left\{u_{i}\right\}=\{1,0\}$, i.e.,

$$
x^{k+1}=\left[\begin{array}{cc}
1 / 2 & 1 / 2 \\
1 / 2 & 1 / 2
\end{array}\right] x^{k}+\left[\begin{array}{l}
1 \\
1
\end{array}\right] u^{k} .
$$

The partition is unsuited as it does not align with any visible system structure. This observation is reflected in $s=\frac{1}{2}$. We embed (22) in an MPC setting with diagonal objectives and box 
TABLE IV

COST-PER-ITERATION OF ALGORITHM 2 FOR THE UNSTRUCTURED SYSTEM (22)

\begin{tabular}{ccccc}
\hline ADMM-type & $M$ & threads & cost & cost/(i) \\
\hline (i) conventional & 1 & 1 & 878 & $100 \%$ \\
(ii) structure-exploiting & $S$ & 1 & 1329 & $151 \%$ \\
(iii) structure-exploiting & $S$ & $2 M N$ & 552 & $63 \%$
\end{tabular}

constraints, which makes the partition admissible. Table IV shows the numerical cost analysis. We see that when we switch from $(i)$ to $(i i)$, the computational cost increases. For a parallel implementation $($ iii $)$, the cost reduces again. However, further simulations show that the overall performance remains worse than for conventional ADMM. Hence, structure exploitation only performs well if the controlled system has structure.

\section{CONClusions And Future Work}

We adapt ADMM to exploit structure in MPC. If the controlled system is sufficiently structured, the resulting algorithm scales well, can be specialized with multiple penalty parameters, is highly parallelizable, and shows improved overall performance. Our algorithm reduces the cost-per-iteration, especially for large and structured systems. The cost reduction comes with an increase in necessary algorithm iterations due to the virtual decomposition of the system. We introduce the separation tendency, a measure of subsystem independence, to relate the iteration increase to the level of structure in the controlled system. Finally, we show a cascade system example, where our structure-exploiting method significantly outperforms conventional ADMM. In future work, the separation tendency can be utilized to determine a partition in the first place. Also, a procedure can be developed that finds a state and input transformation for maximizing exploitable structure, while maintaining the partition admissibility. Furthermore, the concepts of system flow and link usage can be used in other areas of system analysis.

\section{APPENDIX A \\ DETAILS AND PROOFS}

\section{A. Stacked Problem Formulation}

For the objective in (7), we use $\mathcal{Q}_{i}=I_{N} \otimes \operatorname{diag}\left(R_{i}, 0_{\mathrm{w}_{i}}, Q_{i}\right)$, $r_{y_{i}}=\left[r_{u_{i}}^{1} ; 0_{\mathrm{w}_{i} \times 1} ; r_{x_{i}}^{2} ; \ldots ; r_{u_{i}}^{N} ; 0_{\mathrm{w}_{i} \times 1} ; r_{x_{i}}^{N+1}\right], q_{i}=-\mathcal{Q}_{i} r_{y_{i}}$, and $K_{i}=\frac{1}{2} r_{y_{i}} \mathcal{Q}_{i} r_{y_{i}}$, where $\otimes$ is the Kronecker product. We use

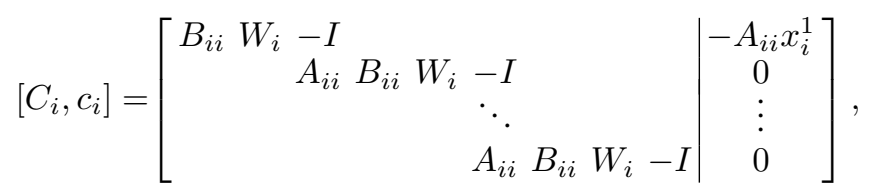

where we understand $C_{i} \in \mathbb{R}^{N \mathrm{x}_{i} \times \mathrm{y}_{i}}$ such that $A_{i i}$ is always below $-I$. For the individual constraints, we choose $\mathcal{Y}_{i}$ such that $\left(u_{i}^{k}, w_{i}^{k}, x_{i}^{k+1}\right) \in \mathcal{U}_{i} \times \mathbb{R}^{\mathrm{w}_{i}} \times \mathcal{X}_{i}$ for all $k$. For the coupling, we recognize that $[\vec{A}, B, W]$ has a row rank defect of $\mathrm{x}-\mathrm{w}$, and we obtain the reduced form $\left[\tilde{A}_{r}, \vec{B}_{r}, W_{r}\right] \in \mathbb{R}^{\mathrm{w} \times(\mathrm{x}+\mathrm{u}+\mathrm{w})}$ by removing linearly dependent rows. We use the reordered variables $\bar{y}=P y$ as in (12) and write (6d) as $\bar{D} \bar{y}=d$ with

$$
[\bar{D}, d]=\left[\begin{array}{lll|l}
B_{r}-W_{r} & & -\ddot{A}_{r} x^{1} \\
& & F & 0_{(N-1) \mathrm{w} \times \mathrm{x}} \\
0
\end{array}\right] \in \mathbb{R}^{N \mathrm{w} \times(\mathrm{y}+1)},
$$

where $F$ is the block-diagonal matrix $I_{N-1} \otimes\left[A_{r}, B_{r},-W_{r}\right]$. We obtain the final form $D y=d$ in $(7 \mathrm{~d})$ with $D=\bar{D} P$.

\section{B. Justification of Algorithm 2 and Proposition 1}

We rewrite the consensus constraint in (9) as

$$
\frac{1}{\sqrt{\rho}} E^{1 / 2}[I ; I] y=\frac{1}{\sqrt{\rho}} E^{1 / 2}[\zeta ; \epsilon],
$$

where $E=\operatorname{diag}\left(E_{\zeta}, E_{\epsilon}\right), E_{\zeta}=\operatorname{diag}\left(E_{\zeta_{1}}, \ldots, E_{\zeta_{M}}\right)$, and $E_{\epsilon}=\operatorname{diag}\left(E_{\epsilon_{1}}, \ldots, E_{\epsilon_{M}}\right)$. The individual scaling matrices are $E_{\zeta_{i}}=\beta \rho_{i} I_{\mathrm{y}_{i}}$ and $E_{\epsilon_{i}}=(1-\beta) \rho_{i} I_{\mathrm{y}_{i}}$. We require $E$ to be positive definite, which ensures that $(23)$ is equivalent to $(9 \mathrm{e})$. Positive definiteness of $E$ is given if and only if $\rho_{i}>0$ and $\beta \in(0,1)$. When we use an augmented Lagrangian as in [8], we obtain

$$
\begin{aligned}
\mathcal{L}_{\rho}\left(y,\left[\begin{array}{l}
\zeta \\
\epsilon
\end{array}\right], \bar{\lambda}\right) & =f(y)+g(\zeta, \epsilon)+\frac{1}{2}\left\|\left[\begin{array}{l}
y \\
y
\end{array}\right]-\left[\begin{array}{l}
\zeta \\
\epsilon
\end{array}\right]-\left[\begin{array}{l}
\bar{\lambda}_{\zeta} \\
\bar{\lambda}_{\epsilon}
\end{array}\right]\right\|_{E}^{2} \\
f(y) & =\sum_{i}\left(\frac{1}{2} y_{i}^{\top} \mathcal{Q}_{i} y_{i}+q_{i}^{\top} y_{i}+\mathcal{I}_{C_{i} y_{i}=c_{i}}\left(y_{i}\right)\right)( \\
g(\zeta, \epsilon) & =\sum_{i} \mathcal{I}_{\mathcal{Y}_{i}}\left(\zeta_{i}\right)+\mathcal{I}_{D \epsilon=d}(\epsilon)
\end{aligned}
$$

where $\bar{\lambda}=\sqrt{\rho} E^{-1 / 2} \lambda,\|x\|_{E}=\left\|E^{1 / 2} x\right\|_{2}=\sqrt{x^{\top} E x}$, and $\mathcal{I}$ denotes an indicator function. By abusing the notation, we replace $\bar{\lambda}$ with $\lambda$. We obtain Algorithm 2 by applying standard ADMM [6, Eqn. (3)] with (24). For $\bar{\Pi}_{D \epsilon=d}(\cdot)$, we obtain

$$
\bar{\Pi}_{D \epsilon=d}(\cdot)=\arg \min _{\epsilon}\left\{\frac{1}{2}\left\|E_{\epsilon}^{1 / 2}(\cdot-\epsilon)\right\|_{2}^{2} \text { s.t. } D \epsilon=d\right\},
$$

which results in (10) through substituting $\bar{\epsilon}=E_{\epsilon}^{1 / 2} \epsilon$. Proposition 1- $(i)$ follows as we have reduced Algorithm 2 to an application of standard ADMM [6]. The algorithm converges to a single fixed point according to [8, Thm. 2], which applies to our formulation as shown in Appendix A-D. Statement $(i i)$ follows from inserting the parameters. We provide additional details on the algorithm formulation in [34].

\section{Justification of Table I}

We denote the cost of an operation with $\mathrm{c}(\cdot)$. Based on (11), the largest subsystem in (2.1) has $\mathcal{O}\left(\max _{i} \mathrm{c}\left(\mathcal{M}_{i}\right)\right)$ as $\mathcal{N}_{i} c_{i}$ can be precomputed. We use $\mathrm{c}\left(\mathcal{M}_{i}\right)=\mathcal{O}\left(\mathrm{c}\left(P_{i}\right)+\mathrm{c}\left(C_{i}\right)+\right.$ $\left.\mathrm{c}\left(C_{i} \mathcal{P}_{i} C_{i}{ }^{-1}\right)\right)$. The cost for multiplying $P_{i}$ relates to $N$-times applying the inverse of $Q_{i}+I$ and $R_{i}+I$, where the first part dominates due to $\mathrm{x} \geq \mathrm{u}$. We precompute an $L D L^{\top}$ factorization and perform a forward-backward substitution as in [47, Sec. 3.1] in $\mathcal{O}\left(N \mathrm{x}_{i}^{2}\right)$. The cost for multiplying $C_{i}$ is dominated by $N$ multiplications with $A_{i i}$, which results in $\mathcal{O}\left(N \mathrm{x}_{i}^{2}\right)$. For $\mathrm{c}\left(C_{i} \mathcal{P}_{i} C_{i}^{-1}\right)$, we again use $L D L^{\top}$, where $L$ is $2 \mathrm{x}_{i}$-banded as the multistage structure makes $C_{i}$ banded. By following [47, Sec. 4.3], we get $\mathcal{O}\left(N \mathrm{x}_{i}^{2}\right)$.

The result for (2.2) follows from the composition of $\mathcal{Y}$ of $M N$ times $\mathcal{X}_{i}, \mathcal{U}_{i}$. In the case of box constraints, the projection reduces to an element-wise clipping in $\mathcal{O}\left(2 \mathrm{x}_{i}+2 \mathrm{u}_{i}\right)=\mathcal{O}\left(\mathrm{x}_{i}\right)$ for each subsystem and time instance. With $2 M N \mathrm{x}_{i}$ parallel threads, (2.2) can also be executed in $\mathcal{O}(1)$.

By following (12), we use $\mathcal{O}(\mathrm{c}(\overline{\mathcal{D}}))$ for (2.3) as we precompute $\overline{\mathcal{E}} d$ and neglect permutations and sums. We use $\mathrm{c}(\overline{\mathcal{D}})=\mathcal{O}\left(\mathrm{c}(\bar{D})+\mathrm{c}\left(\left(\bar{D} \bar{E}_{\epsilon} \bar{D}^{\top}\right)^{-1}\right)\right.$ with $\bar{D}$ from Appendix A-A. $\bar{D}$ is dominated by $N$ diagonal blocks 
$\bar{D}^{k}=\left[\bar{A}_{r}, B_{r},-W_{r}\right]$. For the largest block, we require $\mathcal{O}\left(\max _{k}\left(\mathrm{c}\left(\bar{D}^{k}\right)+\mathrm{c}\left(\left(\bar{D}^{k} \bar{E}_{\epsilon}{ }^{k}\left(\bar{D}^{k}\right)^{\top}\right)^{-1}\right)\right)\right.$. We neglect $\mathrm{c}\left(\bar{D}^{k}\right)$ as $\bar{D}^{k}$ is sparse if the system has structure. For c $\left.\left(\left(\bar{D}^{k} \bar{E}_{\epsilon}{ }^{k}\left(\bar{D}^{k}\right)^{\top}\right)^{-1}\right)\right)$, we use an $L D L^{\top}$ factorization [47, Sec. 3.1] in $\mathcal{O}\left(\mathrm{w}^{2}\right)$. In the 'out-1' case, $A, B$ only have one element per column, hence we can reshuffle $\bar{D}^{k} \bar{E}_{\epsilon}{ }^{k}\left(\bar{D}^{k}\right)^{\top}$ to become block-diagonal. The largest block then has the size $\max _{i} \mathrm{w}_{i}$.

Step (2.4) decomposes into $2 M N$ operations of size $\mathrm{y}_{i}^{k}$. The longest thread has $\mathcal{O}\left(\max _{i}\left\{\mathrm{u}_{i}+\mathrm{w}_{i}+\mathrm{x}_{i}\right\}\right)=\mathcal{O}\left(\max _{i} \mathrm{x}_{i}\right)$. Further, with $2 M N \mathrm{x}_{i}$ parallel threads, it even is $\mathcal{O}(1)$.

\section{Proof of Proposition 2}

Our result extends the convergence analysis in [8] by including a scaled consensus constraint. Instead of $y, w, Q$, $q, A, b, \mathcal{Y}$ as used in [8], we use the notation $\hat{y}, \hat{w}, \hat{Q}, \hat{q}, \hat{A}$, $\hat{b}, \hat{\mathcal{Y}}$, where (9) relates to $[8$, Eqn. (3)] through

$$
\begin{aligned}
\hat{y} & =[\bar{y} ; y], \hat{w}=[\zeta ; \epsilon], \hat{\lambda}=\left[\lambda_{\zeta} ; \lambda_{\epsilon}\right] \\
\hat{Q} & =1 / 2 \operatorname{diag}(\mathcal{Q}, \mathcal{Q}), \text { where } \mathcal{Q}=\operatorname{diag}\left(\mathcal{Q}_{1}, \ldots, \mathcal{Q}_{M}\right) \\
\hat{q} & =1 / 2[q ; q], \text { where } q=\left[q_{1} ; \ldots ; q_{M}\right] \\
\hat{A} & =\left[\begin{array}{cc}
I_{\mathrm{y}} & -I_{\mathrm{y}} \\
0_{N \mathrm{x} \times \mathrm{y}} & C
\end{array}\right], \text { where } C=\operatorname{diag}\left(C_{1}, \ldots, C_{M}\right) \\
\hat{b} & =\left[0_{\mathrm{y} \times 1} ; c\right], \text { where } c=\left[c_{1} ; \ldots ; c_{M}\right] \\
\hat{\mathcal{Y}} & =\left\{\hat{w}=[\zeta ; \epsilon] \mid \zeta_{i} \in \mathcal{Y}_{i}, i=1, \ldots, M, D \epsilon=d\right\} .
\end{aligned}
$$

In [8], it is required that $\{\hat{y} \mid \hat{A} \hat{y}=\hat{b}\} \cap \hat{\mathcal{Y}} \neq \emptyset, \hat{A}$ has full row rank, and $\hat{Z}^{\top} \hat{Q} \hat{Z}$ is positive definite, where $\hat{Z}$ contains an

\begin{tabular}{|c|c|}
\hline Algorithn & 3 ADMM as in [8, Eqn. (5)] \\
\hline repeat & $\begin{array}{l}\text { (3.1. } \hat{y} \leftarrow \hat{\mathcal{M}}\left(\hat{w}+\hat{\lambda}-E^{-1} \hat{q}\right)+\hat{\mathcal{N}} \hat{b} \\
\text { (3.2) } \hat{w} \leftarrow T(\hat{y}-\hat{\lambda}) \\
\text { (3.3) } \hat{\lambda} \leftarrow \hat{\lambda}-\hat{y}+\hat{w}\end{array}$ \\
\hline
\end{tabular}
orthonormal null space basis for $\hat{A}$. With (25), $\hat{Z}=\frac{1}{\sqrt{2}}[Z ; Z]$, and the assumptions made for the initial problem and Proposition 2, these conditions are satisfied. By using a scaling as in (23) and following [8], we obtain Algorithm 3 and the definitions below.

$$
\begin{aligned}
\hat{\mathcal{M}} & =\left[I_{\mathrm{y}} ; I_{\mathrm{y}}\right] \operatorname{diag}\left(\mathcal{M}_{i}\right)\left[\operatorname{diag}\left(\frac{1}{\rho_{i}} E_{\zeta_{i}}\right), \operatorname{diag}\left(\frac{1}{\rho_{i}} E_{\epsilon_{i}}\right)\right] \\
\hat{\mathcal{N}} & =\left[0_{(\mathrm{y}+N \mathrm{x}) \times \mathrm{y}} \operatorname{diag}\left(\mathcal{N}_{i}\right)\right] \\
T(\cdot) & =E^{-1 / 2} \Pi_{E^{1 / 2}} \hat{\mathcal{Y}}\left(E^{1 / 2} \cdot\right)
\end{aligned}
$$

In (26), we use $\mathcal{M}_{i}$ and $\mathcal{N}_{i}$ as in (11). To be consistent with [8], we require $(i)$ that $\hat{\mathcal{M}}$ is a contraction, and $(i i)$ that $T(\cdot)$ is firmly nonexpansive [48], which is equivalent to the properties noted in [8, Eqn. (8), Lem. 3] by [48, Definition 4.1, Proposition 4.2]. For $(i)$, it is sufficient to check if all $\mathcal{M}_{i}$ are contractions. Similar to [8, Eqn. (5)], we use the null space method in [32, Sec. 16.2] to obtain the equivalent form $\mathcal{M}_{i}=Z_{i}\left(Z_{i}^{\top}\left(\frac{1}{\rho_{i}} Q_{i}+I\right) Z_{i}\right)^{-1} Z_{i}^{\top}$. It can be shown that $(i)$ is satisfied if $\hat{Z}_{i}^{\top} \hat{Q}_{i} \hat{Z}_{i}$ is positive definite. Requirement (ii) is true by [48, Proposition 4.8] as $T(\cdot)$ is an orthogonal projection onto a convex set in a Hilbert space with inner product $\langle x, y\rangle_{E}=x^{\top} E y$. As $(i),(i i)$ are satisfied, the convergence analysis in [8] applies. In [8, Sec. V], the worst-case convergence rate is optimized with $\rho^{\star}=\arg \min _{\rho}\|\tilde{\mathcal{M}}\|_{2}$, where $\tilde{\mathcal{M}}=\hat{Z}^{\top} \hat{\mathcal{M}} \hat{Z}-\frac{1}{2} I_{N \mathrm{x}}$, which becomes $\tilde{\mathcal{M}}=\operatorname{diag}\left(Z_{i}^{\top} \mathcal{M}_{i} Z_{i}-\frac{1}{2} I_{N \mathrm{x}_{i}}\right)$ in our case. As $\tilde{\mathcal{M}}$ is block-diagonal, we choose separate $\rho_{i}$ in the same way as $\rho$ is chosen in [8], which results in Proposition 2. We show a more-detailed version of this proof in [34].

\section{E. Proof of Proposition 3}

For $(i)$, we show $0<\sum_{j} \Gamma_{i j}<\infty$ for all $i$. First, we consider (1b) with $u^{k}=\delta^{k}, x^{0}=0$. We obtain $x^{k}=(A)^{k-1} B 1_{\mathrm{u} \times 1}$ for $k \geq 1$. Given that $A$ is semi-convergent, $x^{k}$ asymptotically converges to $x_{e q}=A x_{e q}$, which implies that $\Delta x^{k}$ asymptotically converges to zero. Hence, $\sum_{k=0}^{\infty}\left|\Delta x^{k}\right|^{2}$ is a sum over a squared-exponential tail, which is finite [49, Eq. (2151), p.1132]. Consequently, each $\Gamma_{i j}$ is finite and $\sum_{j} \Gamma_{i j}<\infty$ for all $i$. To show $\sum_{j} \Gamma_{i j}>0$, we use that controllability implies that $\left[B, A B, A^{2} B, \ldots, A^{\mathrm{x}-1} B\right]$ has full rank, which means that the sequence $\left\{x^{k}\right\}=\left\{A^{k-1} B 1_{\mathrm{u} \times 1}\right\}$ spans $\mathbb{R}^{\mathrm{x}}$. The same is true for $\left\{\Delta x^{k}\right\}$. Also, controllability implies that $A, B$ do not have a common zero row. Hence, no row in $\Phi^{k}$ is filled with zeros for all times, and we obtain $\sum_{j} \Gamma_{i j}>0$ for all $i$.

Statement (ii) is clear from $0<\sum_{j} \Gamma_{i j}<\infty$ and (18a).

For (iii), we use the diagonal state and input transformation $T=\operatorname{diag}\left(t_{1}, t_{2}, \ldots, t_{\mathrm{x}+\mathrm{u}}\right)$ with $t_{i} \neq 0$. More specifically, we use $\left[\bar{x}^{k}, \bar{u}^{k}\right]=T^{-1}\left[x^{k}, u^{k}\right]$. For the transformed system, we obtain $\bar{\Phi}^{k}=T^{-1} \Phi^{k}$ and $\bar{\Gamma}_{i j}=\left|t_{i}\right| \Gamma_{i j}$. The factors $\left|t_{i}\right|$ then cancel out in (18a), which makes $s$ invariant to $T$.

\section{REFERENCES}

[1] J. M. Maciejowski, Predictive Control with Constraints. Prentice Hall, 2002.

[2] E. F. Camacho and C. B. Alba, Model predictive control. Springer Science \& Business Media, 2013.

[3] M. Morari and J. H. Lee, "Model predictive control: past, present and future," Computers \& Chemical Engineering, vol. 23, no. 4-5, pp. 667682, 1999.

[4] S. Di Cairano, "An industry perspective on MPC in large volumes applications: Potential benefits and open challenges," IFAC Proceedings Volumes, vol. 45, no. 17, pp. 52-59, 2012.

[5] R. Glowinski and A. Marroco, "Sur l'approximation, par éléments finis d'ordre un, et la résolution, par pénalisation-dualité d'une classe de problèmes de dirichlet non linéaires," Revue française d'automatique, informatique, recherche opérationnelle. Analyse numérique, vol. 9, no. 2, pp. 41-76, 1975.

[6] S. Boyd, N. Parikh, E. Chu, B. Peleato, and J. Eckstein, "Distributed optimization and statistical learning via the alternating direction method of multipliers," Foundations and Trends in Machine Learning, vol. 3, no. 1, pp. 1-122, 2011.

[7] J. L. Jerez, P. J. Goulart, S. Richter, G. A. Constantinides, E. C. Kerrigan, and M. Morari, "Embedded online optimization for model predictive control at megahertz rates," IEEE Transactions on Automatic Control, vol. 59, no. 12, pp. 3238-3251, 2014.

[8] A. U. Raghunathan and S. Di Cairano, "Optimal step-size selection in alternating direction method of multipliers for convex quadratic programs and model predictive control," in Proceedings of Symposium on Mathematical Theory of Networks and Systems, 2014, pp. 807-814.

[9] J. L. Jerez, P. J. Goulart, S. Richter, G. A. Constantinides, E. C. Kerrigan, and M. Morari, "Embedded predictive control on an FPGA using the fast gradient method," in European Control Conference. IEEE, 2013, pp. 3614-3620.

[10] H. Peyrl, A. Zanarini, T. Besselmann, J. Liu, and M.-A. Boéchat, "Parallel implementations of the fast gradient method for high-speed MPC," Control Engineering Practice, vol. 33, pp. 22-34, 2014. 
[11] M. G. Vayá, G. Andersson, and S. Boyd, "Decentralized control of plugin electric vehicles under driving uncertainty," in Innovative Smart Grid Technologies Conference Europe. IEEE, 2014, pp. 1-6.

[12] J. Kang, A. U. Raghunathan, and S. Di Cairano, "Decomposition via ADMM for scenario-based model predictive control," in American Control Conference. IEEE, Jul 2015, pp. 1246-1251.

[13] F. Rey, X. Zhang, S. Merkli, V. Agliati, M. Kamgarpour, and J. Lygeros, "Strengthening the group: Aggregated frequency reserve bidding with admm," IEEE Transactions on Smart Grid, 2018.

[14] W. Deng, M.-J. Lai, Z. Peng, and W. Yin, "Parallel multi-block ADMM with o(1/k) convergence," Journal of Scientific Computing, vol. 71, no. 2, pp. 712-736, 2017.

[15] C. Chen, B. He, Y. Ye, and X. Yuan, "The direct extension of ADMM for multi-block convex minimization problems is not necessarily convergent," Mathematical Programming, vol. 155, no. 1-2, pp. 57-79, 2016.

[16] G. Mateos, J. A. Bazerque, and G. B. Giannakis, "Distributed sparse linear regression," IEEE Transactions on Signal Processing, vol. 58, no. 10 , pp. $5262-5276,2010$

[17] T.-H. Chang, M. Hong, and X. Wang, "Multi-agent distributed optimization via inexact consensus ADMM," IEEE Transactions on Signal Processing, vol. 63, no. 2, pp. 482-497, 2015.

[18] B. Stellato, G. Banjac, P. Goulart, A. Bemporad, and S. Boyd, "OSQP: An operator splitting solver for quadratic programs," arXiv preprint arXiv:1711.08013, 2017.

[19] A. U. Raghunathan and S. Di Cairano, "ADMM for convex quadratic programs: Q-linear convergence and infeasibility detection," arXiv preprint arXiv:1411.7288, 2014.

[20] E. Ghadimi, A. Teixeira, I. Shames, and M. Johansson, "Optimal parameter selection for the alternating direction method of multipliers (ADMM): Quadratic problems," IEEE Transactions on Automatic Control, vol. 60, no. 3, pp. 644-658, Mar 2015.

[21] F. Rey, D. Frick, A. Domahidi, J. Jerez, M. Morari, and J. Lygeros, "ADMM prescaling for model predictive control," in Conference on Decision and Control. IEEE, 2016, pp. 3662-3667.

[22] G. Stathopoulos, H. Shukla, A. Szucs, Y. Pu, C. N. Jones et al., "Operator splitting methods in control," Foundations and Trends in Systems and Control, vol. 3, no. 3, pp. 249-362, 2016.

[23] P. Giselsson and S. Boyd, "Linear convergence and metric selection for douglas-rachford splitting and ADMM," IEEE Transactions on Automatic Control, vol. 62, no. 2, pp. 532-544, 2017.

[24] Y. Wang and S. Boyd, "Fast model predictive control using online optimization," IEEE Transactions on Control Systems Technology, vol. 18, no. 2, pp. 267-278, 2010.

[25] F. Rey, P. Hokayem, and J. Lygeros, "A tailored ADMM approach for power coordination in variable speed drives," 20th IFAC World Congress, vol. 50, no. 1, pp. 7403-7408, 2017.

[26] _ , "Ask not what ADMM can do for you, ask what you can do for ADMM - virtual subsystems in MPC," in Conference on Decision and Control. IEEE, 2017, pp. 4357-4362.

[27] A. A. Ageev and M. I. Sviridenko, "An approximation algorithm for hypergraph max k-cut with given sizes of parts," in European Symposium on Algorithms. Springer, 2000, pp. 32-41.

[28] A. Frieze and M. Jerrum, "Improved approximation algorithms for maxk-cut and max bisection," Algorithmica, vol. 18, no. 1, pp. 67-81, 1997.

[29] J. P. Hespanha, "An efficient matlab algorithm for graph partitioning," University of California, pp. 1-8, 2004.

[30] N. Parikh, S. Boyd et al., "Proximal algorithms," Foundations and Trends in Optimization, vol. 1, no. 3, pp. 127-239, 2014.

[31] G. Banjac, P. Goulart, B. Stellato, and S. Boyd, "Infeasibility detection in the alternating direction method of multipliers for convex optimization," Optimization Online, 2017.

[32] J. Nocedal and S. J. Wright, Numerical optimization. Springer Science and Business Media, 1975, vol. 9, no. 4

[33] A. Domahidi, A. U. Zgraggen, M. N. Zeilinger, M. Morari, and C. N. Jones, "Efficient interior point methods for multistage problems arising in receding horizon control," in Conference on Decision and Control. IEEE, 2012, pp. 668-674.

[34] F. Rey, P. Hokayem, and J. Lygeros, "ADMM for exploiting structure in MPC," arXiv preprint arXiv:1808.06879, Aug 2018.

[35] Y. Okuyama, Discrete control systems. Springer, 2014.

[36] K. Alyani, M. Congedo, and M. Moakher, "Diagonality measures of hermitian positive-definite matrices with application to the approximate joint diagonalization problem," Linear Algebra and its Applications, vol. 528, pp. 290-320, 2017.

[37] R. Toscano, Structured controllers for uncertain systems. Springer, 2013.
[38] A. V. Oppenheim, Discrete-time signal processing. Pearson Education India, 1999.

[39] G. Gu, Discrete-Time Linear Systems: Theory and Design with Applications. Springer Science \& Business Media, 2012.

[40] M. Cantoni, F. Farokhi, E. Kerrigan, and I. Shames, "Structured computation of optimal controls for constrained cascade systems," International Journal of Control, pp. 1-10, 2017.

[41] Y. Li, M. Cantoni, and E. Weyer, "On water-level error propagation in controlled irrigation channels," in Conference on Decision and Control. IEEE, 2005, pp. 2101-2106.

[42] L. Soltanian and M. Cantoni, "Decentralized string-stability analysis for heterogeneous cascades subject to load-matching requirements," Multidimensional Systems and Signal Processing, vol. 26, no. 4, pp. 985-999, 2015.

[43] J. W. Labadie, "Optimal operation of multireservoir systems: state-ofthe-art review," Journal of water resources planning and management, vol. 130, no. 2, pp. 93-111, 2004.

[44] G. Guo and W. Yue, "Hierarchical platoon control with heterogeneous information feedback," IET control theory \& applications, vol. 5, no. 15, pp. 1766-1781, 2011

[45] J. Lofberg, "YALMIP: A toolbox for modeling and optimization in MATLAB," in International Symposium on Computer Aided Control Systems Design. IEEE, 2004, pp. 284-289.

[46] I. Gurobi Optimization, "Gurobi optimizer reference manual," 2016. [Online]. Available: http://www.gurobi.com

[47] G. H. Golub and C. F. Van Loan, Matrix Computations. Baltimore, MD, USA: Johns Hopkins University Press, 1996.

[48] H. H. Bauschke and P. L. Combettes, Convex analysis and monotone operator theory in Hilbert spaces. Springer, 2017, vol. 2011.

[49] I. N. Bronstein, J. Hromkovic, B. Luderer, H.-R. Schwarz, J. Blath, A. Schied, S. Dempe, G. Wanka, and S. Gottwald, Taschenbuch der mathematik. Springer-Verlag, 2012, vol. 1.

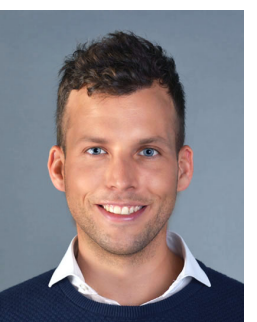

Felix Rey received his B.Eng. degree from the University of Applied Sciences Constance (Germany, 2011), and M.Sc. degree from the Karlsruhe Institute of Technology (Germany, 2014), both in Electrical Engineering and Information Technology. $\mathrm{He}$ is a fellow of the German National Academic Foundation. In 2014, he joined the Automatic Control Laboratory at ETH Zurich as a Ph.D. candidate. His research interests include model predictive control, as well as distributed and embedded optimization, in particular with ADMM.

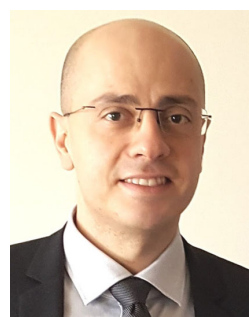

Peter Hokayem received his B.Eng. degree in Computer and Communications Engineering from the American University of Beirut in 2001, his M.Sc. degree in Electrical Engineering from the University of New Mexico in 2003, and his Ph.D. degree in Electrical and Computer Engineering from the University of Illinois at Urbana-Champaign in 2007. Following a Postdoctoral Scholar position at the University of Wuerzburg, he joined the Automatic Control Laboratory at ETH Zurich in 2008 as a (Senior) Postdoctoral Researcher. He moved to ABB Switzerland in 2011 and since then has held several positions, both at the Business Unit and the Corporate Research Center. Currently, he has a consulting role at $\mathrm{ABB}$ as a Drive System Expert in Medium Voltage Drives, with a strong focus on novel estimation and control methods for largescale electromechanical power conversion systems. He was a recipient of the Automatica Paper Prize Award in 2008 and is a Senior Member of the IEEE. 


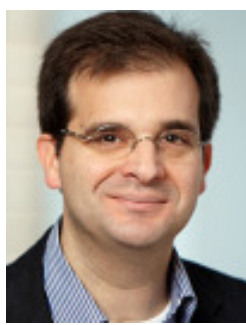

John Lygeros (M'90-F'11) received his B.Eng. degree in electrical engineering (1990) and his M.Sc. degree in Systems and Control (1991), both at Imperial College of Science Technology and Medicine, London, UK, and his Ph.D. degree from the Electrical Engineering and Computer Sciences Department, University of California, Berkeley (1996). He holds the chair of Computation and Control at the Swiss Federal Institute of Technology (ETH) Zurich, Switzerland, where he is currently serving as the Head of the Automatic Control Laboratory. He held a series of research appointments at the National Automated Highway Systems Consortium, Berkeley, the Laboratory for Computer Science, M.I.T., and the Electrical Engineering and Computer Sciences Department at U.C. Berkeley (1996-2000). He was a University Lecturer at the Department of Engineering, University of Cambridge, UK, and a Fellow of Churchill College (2000-2003). He was an Assistant Professor at the Department of Electrical and Computer Engineering, University of Patras, Greece (2003-2006). In 2006 he joined the Automatic Control Laboratory at ETH Zurich, first as an Associate Professor, and since 2010 as Full Professor. His research interests include modeling, analysis, and control of hierarchical, hybrid, and stochastic systems, with applications to biochemical networks, automated highway systems, air traffic management, power grids, and camera networks. He is a Fellow of the IEEE and a Member of the IET and the Technical Chamber of Greece. Since 2013, he is serving as the Treasurer and a Council Member of the International Federation of Automatic Control. 